\title{
Noninvasive Remote Sensing Techniques for Infrastructures Diagnostics
}

\author{
Angelo Palombo, ${ }^{1}$ Stefano Pignatti, ${ }^{1}$ Angela Perrone, ${ }^{1}$ Francesco Soldovieri, ${ }^{2}$ \\ Tony Alfredo Stabile, ${ }^{3}$ and Simone Pascucci ${ }^{1}$ \\ ${ }^{1}$ Consiglio Nazionale delle Ricerche, Istituto di Metodologie per l'Analisi Ambientale (IMAA), 85050 Tito Scalo, Italy \\ ${ }^{2}$ Consiglio Nazionale delle Ricerche, Istituto per il Rilevamento Elettromagnetico dell'Ambiente (IREA), 80127 Naples, Italy \\ ${ }^{3}$ Dipartimento di Scienze Fisiche, Università degli Studi di Napoli Federico II, 80126 Naples, Italy
}

Correspondence should be addressed to Angelo Palombo, palombo@imaa.cnr.it

Received 15 February 2011; Revised 15 April 2011; Accepted 25 May 2011

Academic Editor: Nicola Masini

Copyright (C) 2011 Angelo Palombo et al. This is an open access article distributed under the Creative Commons Attribution License, which permits unrestricted use, distribution, and reproduction in any medium, provided the original work is properly cited.

\begin{abstract}
The present paper aims at analyzing the potentialities of noninvasive remote sensing techniques used for detecting the conservation status of infrastructures. The applied remote sensing techniques are ground-based microwave radar interferometer and InfraRed Thermography (IRT) to study a particular structure planned and made in the framework of the ISTIMES project (funded by the European Commission in the frame of a joint Call "ICT and Security" of the Seventh Framework Programme). To exploit the effectiveness of the high-resolution remote sensing techniques applied we will use the high-frequency thermal camera to measure the structures oscillations by high-frequency analysis and ground-based microwave radar interferometer to measure the dynamic displacement of several points belonging to a large structure. The paper describes the preliminary research results and discusses on the future applicability and techniques developments for integrating high-frequency time series data of the thermal imagery and ground-based microwave radar interferometer data.
\end{abstract}

\section{Introduction}

During the last decades, the advances in nondestructive electromagnetic sensing techniques (optical, thermal, and microwave) operating at different spectral, spatial and time scales make a multidepth, multiresolution and multiscale diagnostics and monitoring activities thus to be used for the status assessment of existing structures [1].

These nondestructive techniques are primarily suited for the detection and characterisation of alterations and defects in the near surface of structures [2] and for the displacement monitoring and verification of the structural integrity of buildings and structures $[3,4]$.

As regards the sensing techniques used for this study, the thermal camera technology is a non-destructive investigation technique commonly used in different applications and in particular in the infrastructures and architectural heritage diagnostics [5]. Thermal imagery is used to identify subsurface ununiformities since the heat flow from the surface to the inner structure is affected by the defects and inhomogeneities present in the structure. The InfraRed Termography (IRT) analysis allows diagnostic results ranging from qualitative characterization (e.g., detection) to quantitative analysis (e.g., inhomogeneities characterization). It is worth noting that the application of IRT for detecting and characterizing defects in construction materials can be quite complicated as different physical properties affecting the thermal and optical properties of the structure [6] have to be considered. Among them, the most important are the conductivity, the diffusivity, and the specific heat of the investigated material, whereas the spectral properties such as emissivity, absorption, reflection, and transmission play a key role on the heat distribution in the material. Other properties/ characteristics that influence the thermal behaviour of a material are related to its porosity, volumetric mass, and water content.

Furthermore, the use of high-frequency imagery thermal camera gives the opportunity of measuring full field 
deformation and vibration characteristics (i.e., frequency and attenuation). From these measurements different modal parameters of the structure (e.g., natural frequencies, mode shapes, and damping ratios) can be extracted and displayed and used for the definition of a baseline set of dynamic characteristics of the structure. This kind of analysis can be subsequently used for the structural health monitoring and the application of vibration-based damage detection techniques (see e.g., $[7,8]$ ).

For the present study, we used high-frequency imagery (HFI) acquisitions for the retrieving of fast displacements and vibrations in the structure.

On the other hand, concerning the microwave interferometry technology applied in this study, it has proven to be a powerful remote sensing tool for vibration measurement of structures [9]. It was recently applied for tracking the vibration of bridges excited by vehicular traffic $[8,10]$ to monitor the displacements of heritage architectural structures such as the two bell towers,

Giotto's Tower and Arnolfo's Tower, in Florence [11] or the Tower of Pisa [12], for deflection measurements on vibrating stay cables [13], and for in-field dynamic monitoring of engineering structures $[4,14]$. All these studies have been carried out by the use of an innovative noncontact microwave interferometer, named IBIS-S (Image By Interferometric Survey of Structures), manufactured by the Italian company IDS S.p.A. This innovative radar system has been also applied for this study and it is based on the Stepped-Frequency Continuous Wave (SF-CW) technique [15] and on the Differential Interferometric technique [16]. The former providing the system with a range resolution capability and the latter allowing the system to evaluate the displacement response of each target detected in the illuminated scenario. IBIS-S sensor is fast and easy to be installed, allowing to operate in all weather conditions, both day and night, even at a significant distance and without the need of installing and wiring sensors. A further advantage obtained by the IBIS-S instrument is the direct measurement of the displacement of a large number of targets simultaneously, in real-time, and with high accuracy.

In this paper, the application of different sensors and methodologies to several noninvasive infrastructures near surface diagnostics is presented. The analysis of acquired data on different structures and materials included also time series recorded by the thermal camera and radar on several points of a test bed cement beam.

\section{Data and Methods}

2.1. Test Beds. The data for this study were acquired in Montagnole (French Alpes) site in an innovative facility owned by LCPC (Laboratoire Central des Ponts et Chaussées), that is one of the ISTIMES partners.

The Montagnole test site is mainly used to certify metallic protection nets that are used in mountains to prevent catastrophic rockslides. For ISTIMES project, it was exploited as a purely research-oriented facility to verify the behaviour of noninvasive sensing techniques during the progressive damage of an on purpose built concrete beam structure. In particular, the experiment has regarded the progressive damage, in different stages, of a concrete beam by means of falling blocks thus allowing the different techniques to be tested in the presence of hazards.

In the Montagnole experiment an iron ball of 2.5 Tons was used (Figure 1(a)) and for this study we analyze only the four falling actions where the ball has impacted directly on the beam. In particular, drops 1 to 3 have been performed from an altitude of $1 \mathrm{~m}$ with respect to the beam and drop 4 from a $5 \mathrm{~m}$ of height. The last drop (i.e., number 4) was used to study and diagnose the significant structural damage as induced by a heavy direct impact of the ball onto the armed concrete beam in order to understand the progressive energy release. Therefore, for this experiment, we assumed that such drop rate was convenient to have a refined monitoring of the beam at the beginning and at the end of each daily experiment session.

2.2. Data. For this study we used the following instrumentation for a rapid and noninvasive diagnostic of the infrastructure status. Specifically, as regards the IRT instrumentation, we used a FLIR SC7900-VL thermal camera (LWIR; 7.7$11.5 \mu \mathrm{m}$ ), whereas an IBIS-S microwave radar interferometer operating in the Ku frequency band with a central frequency of $16.75 \mathrm{GHz}$ was used for the microwave interferometry.

The FLIR camera features has a "snap shot" integration range from $10 \mu$ s to $10 \mathrm{~ms}$, which incorporates a high quantic efficiency MCT focal plane array thus ensuring a very highspectral resolution with a Noise Equivalent Temperature Difference (NEDT) $<25 \mathrm{mK}$. The frame rates are up to 200 FPS (frames per second) in full frame mode $(320 \times 256)$ and 6000 FPS in subwindowing mode. In this study, we used the following optimal configuration of the LWIR camera: $160 \times 128$ frames, thus allowing 800 FPS.

The IBIS-S microwave radar consists of a sensor module installed on a tripod with a 3D rotating head. An USB interface allows the connection with a control PC, which is used to configure the acquisition parameters, to view in real time the first results, and to perform the storage of the measurements. The two horn antennas transmit the electromagnetic signals in the frequency band of $16.75 \pm 0.30 \mathrm{GHz}$ and receive the echoes from the target. Sensor module and PC are connected to a battery unit.

IBIS-S system can provide a range resolution of $0.5 \mathrm{~m}$, a displacement sensitivity of $0.01 \mathrm{~mm}$, a maximum operational distance of $500 \mathrm{~m}$, and a maximum sampling frequency of $200 \mathrm{~Hz}$ [17].

\subsection{Methods}

2.3.1. HFI Thermal Imagery. In the survey of measurements on the Montagnole test site, we took advantage of the thermal camera (LWIR) to obtain very high-frequency thermal imagery on the concrete beam during the direct impact of the falling blocks. The FLIR camera was mounted on a tripod at about $40 \mathrm{~m}$ (i.e., in box 3) and $70 \mathrm{~m}$ (i.e., near box 5) of distance from the beam. Box 3 was used for the first three 


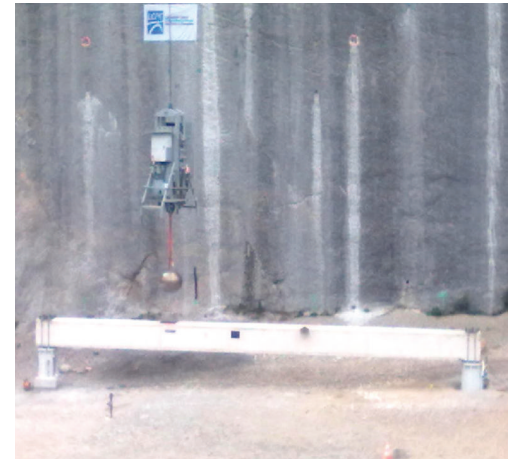

(a)

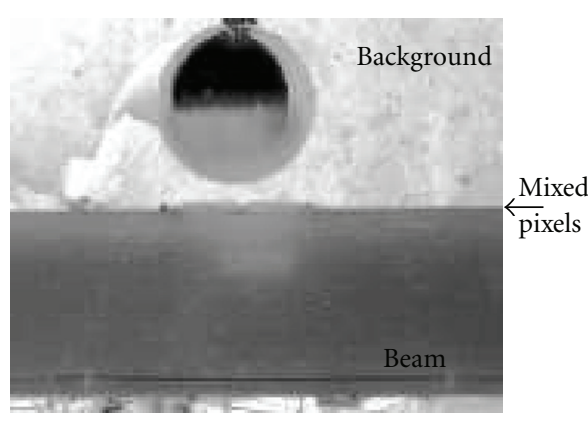

(b)

Figure 1: (a) Picture of the experimental site with the heavy load (iron ball) suspended at the height of $1 \mathrm{~m}$ over the beam. (b) Example of a frame acquired by HFI FLIR camera. The frame depicts the heavy load just before the impact on the concrete beam. The black arrow indicates the mixed pixels (i.e., background and beam) used for calculating the oscillation frequency after the impact.

drops (height of the falling ball of $1 \mathrm{~m}$ ); for the drop 4 (height of the falling ball of $5 \mathrm{~m}$ ), due to safety reasons, the camera was installed near the box $5(70 \mathrm{~m})$. For all the drops, the acquisition time was of $46 \mathrm{~s}$ for a total of 37000 frames.

The thermal imagery acquisitions were used to calculate the beam oscillation frequency as derived from the difference between the beam temperature and the background temperature (see Figure 1(b)).

The method is based on the presence of mixed pixels composed by both the beam and the background as shown by the black arrow in Figure 1(b), labelled as $i$-pixel. The $i$ pixel temperature measured by the FLIR camera accounts for the time-varying temperature relative to the beam and the background. The following equations were used to retrieve the beam oscillation:

$$
\begin{gathered}
c_{1} T_{b}+c_{2} T_{t}=T_{m}, \\
c_{1}+c_{2}=1,
\end{gathered}
$$

where, $c_{1}$ and $T_{b}$ are the fraction of the $i$-pixel occupied by the background and its temperature, respectively; $c_{2}$ and $T_{t}$ are the fraction of the $i$-pixel occupied by the beam and its temperature, respectively. $T_{m}$ is the temperature measured by the camera for the $i$-pixel.

The first equation of (1) represents the linear mixing of the temperatures of the beam and the background, the second is the constrain used for the total pixel fraction. The $T_{b}$ and $T_{t}$ temperatures, which are unknowns in (1), can be extracted from the temperature values of the neighbouring pixels of the $i$-pixel. The neighbouring pixels used for calculating $T_{b}$ and $T_{t}$ have to be pure pixels (i.e., made up of only beam or target).

In (1), $c_{2}$ represents the oscillation quantity expressed in terms of pixel fraction that varies from 0 to 1 . Moreover, when $c_{2}$ oscillation reaches values near to the maximum and minimum, this represents situations in which the amplitude of oscillation is close to or greater than the maximum measurable for a pixel, respectively. In the case that a pixel is not sufficient to encompass the oscillation, it is possible to solve the problem by considering, using the sum of the neighbouring pixels, "equivalent pixels" having a larger dimension compared to the original one.

\subsubsection{Ground-Based Microwave Radar Interferometer. IBIS-S} is based on two well-known radar techniques: the SteppedFrequency Continuous Wave (SF-CW) and the Differential Interferometric $[12,18]$.

The SF-CW technique allows the system to resolve the scenario in the range direction $[15,19]$, that is, to detect the position of target surfaces placed at different distances from the sensor. It is based on the synthesis and transmission of a burst of $N$ monochromatic pulses equally and incrementally spaced in frequency (with fixed frequency step of $\Delta f$ ) leading to a work bandwidth $B$ :

$$
B=(N-1) \Delta f,
$$

The $N$ monochromatic pulses sample the scenario in the frequency domain similarly to a short pulse with a large bandwidth $B$. In a SF-CW radar, the signal source dwells at each frequency $f_{x}=f_{0}+k \Delta f(k=0,1,2, \ldots, N-1)$, the single tone signal is sufficiently long to allow the echoes backscattered by the target to reach the receiver. Hence, the duration of each monochromatic pulse $\left(T_{\text {pulse }}\right)$ depends on the maximum distance $\left(R_{\max }\right)$ to be observed in the scenario and is given by,

$$
T_{\text {pulse }} \geq \frac{2 R_{\max }}{c},
$$

where $c$ represents the velocity of the light.

At each sampled time instant, both $I$ (In-phase) and Q (Quadrature) components of the received signals are acquired so that the resulting data consist of a vector of $N$ complex samples, representing the frequency response measured at $N$ discrete frequencies.

By taking the Inverse Discrete Fourier Transform (IDFT) the radar response is reconstructed in the time domain: each complex sample in this domain represents the signal (echo) from a range (distance) interval of length $c T_{\text {pulse }} / 2$.

The amplitude range profile of the radar echoes is then obtained by calculating the magnitude of each bin of 


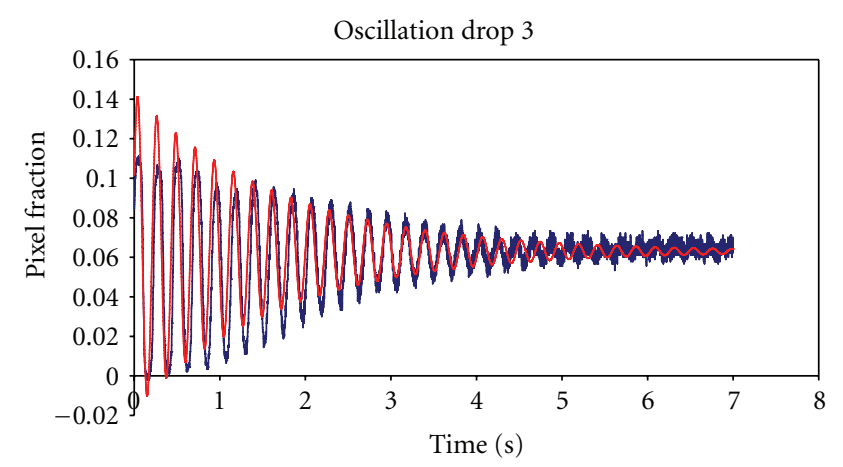

— Pixel fraction shot 3

- Dumper oscillator

Figure 2: The graph shows the under-dumping decay attained for the fraction of the $i$-pixel occupied by the beam (i.e., blue line $C_{2}$ ) used for the Montagnole experiment. Red line depicts the theoretical dumper oscillator calculated from the parameters of Table 1.

the IDFT of acquired vector samples. This range profile gives a one-dimensional mapping of scattering objects in the "view space" as function of their relative distance from the sensor.

The differential interferometric technique allows the system to measure the displacements of the structure illuminated by the antenna main beam by comparing the phase information of the backscattered electromagnetic waves collected at different times (see, e.g., [16]).

Generally speaking, when a target surface moves with respect to the sensor module (emitting and back-receiving the electromagnetic wave) at least a phase shift arises between the signals reflected by the target surface at different times. Hence, the displacement of the investigated object is determined from the phase shift measured by the radar sensor at the discrete acquisition times. The radial displacement $d_{p}$ (i.e., the displacement along the direction of wave propagation) and the phase shift $\Delta \varphi$ are linked by the following:

$$
d_{p} \propto \frac{\lambda}{4 \pi} \Delta \varphi
$$

where $\lambda$ is the wavelength of the probing electromagnetic signal.

The radar emits a series of electromagnetic waves for the entire measurement period and processes phase information at regular time intervals to find any displacement occurring between one emission and the next one. It is worth underlining that the interferometric technique in (4) provides a measurement of the displacement along the line of sight for all the range bins of the structure illuminated by the antenna beam; once $d_{p}$ has been evaluated, the vertical displacement $d$ can be easily found by making some geometric projections.

IBIS-S system was applied in both Montagnole and Musmeci test beds. In the first case it was installed on a hill at a height of about $15 \mathrm{~m}$ from the ground level and at a distance of about $70 \mathrm{~m}$ from the target. In order to improve the accuracy and the signal-to-noise ratio of the measurements, a corner reflector was fixed on the beam. To acquire the measurements a range sampling frequency of $200 \mathrm{~Hz}$ and an acquisition time of about $60 \mathrm{~s}$ for each drop were used.

In the Musmeci test bed IBIS-S system was placed just under the bridge deck with a perpendicular direction at a distance of about $30 \mathrm{~m}$. A sampling frequency of about $100 \mathrm{~Hz}$ and an acquisition time of about 5 minutes were applied.

In both cases the use of IBIS-S system allowed the dynamic monitoring of the investigated target without any contact between the sensor and the investigated target. This characteristic was very useful in Montagnole test bed where very restricted security conditions were required in order to carry out the experiment.

\section{Results and Discussion}

3.1. HFI Results. For Montagnole experiment, the drops of the heavy iron ball on the beam were used as mechanical solicitation to measure the dynamic response of the beam. For this study we used a simple model by considering the behaviour of the beam as the one of a damped harmonic oscillator. The equation describing the oscillation is

$$
z(t)=A_{0} e^{-\varsigma \omega_{0} t} \sin \left(\sqrt{1-\varsigma} \omega_{0} t+\varphi\right),
$$

where $A_{0}$ is the amplitude and $\varphi$ the phase that dictates the initial conditions, $\omega_{0}$ is the undamped angular frequency of the oscillator and $\zeta$ is damping ratio that are given, respectively, by:

$$
\begin{aligned}
& \omega_{0}=\sqrt{\frac{k}{m}}, \\
& \varsigma=\frac{c}{2 m \omega_{0}},
\end{aligned}
$$

where $k$ is the elastic constant, $m$ is the mass, and $c$ is the viscous damping coefficient.

The value of $\zeta$ determines the behaviour of the system in fact, the damped harmonic oscillator can be (a) for $\zeta>1$, the system is represented by exponentially decays; (b) for $\zeta=$ 1 , the system returns to equilibrium as quickly as possible without oscillating; (c) for $\zeta<1$, the system is represented by an underdamped harmonic oscillator (Figure 2).

The quantity that describes the beam oscillation is the $c_{2}$ value represented in (1). This value was calculated for all the frames of each acquisition performed on the beam. Figure 2 depicts the comparison between the quantity $c_{2}$ (blue line) calculated for the third drop and the theoretical underdamped harmonic oscillator (red line) that approximates the $c_{2}$ oscillations. The parameters used for evaluating the underdamped harmonic oscillation, that is, those that better approximates the $c_{2}$ oscillation, are reported in Table 1 .

Moreover, in order to obtain the resonance frequencies of the beam we applied the Fast Fourier Transform (FFT) on the quantity $c_{2}$ evaluated for the four drops. Figure 3 depicts the time behaviour of the $c_{2}$ for the four drops, whereas Figure 4 reports the amplitude of the FFT of $c_{2}$. Table 2 shows the oscillation frequencies of the two most significant FFT coefficients. 


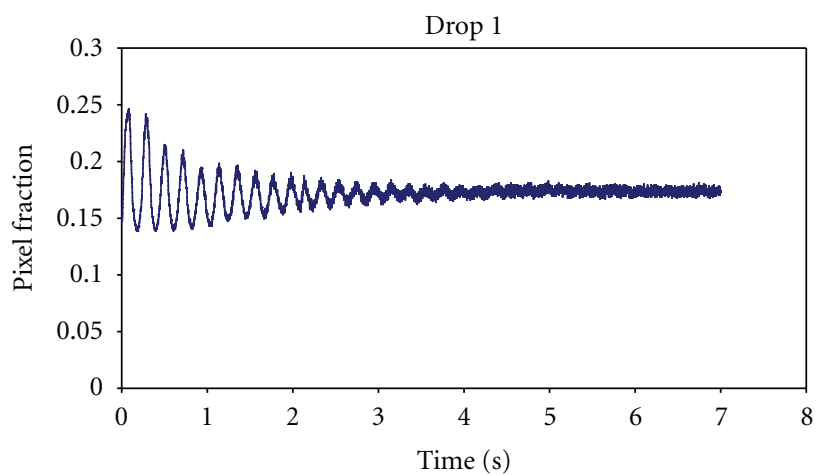

(a)

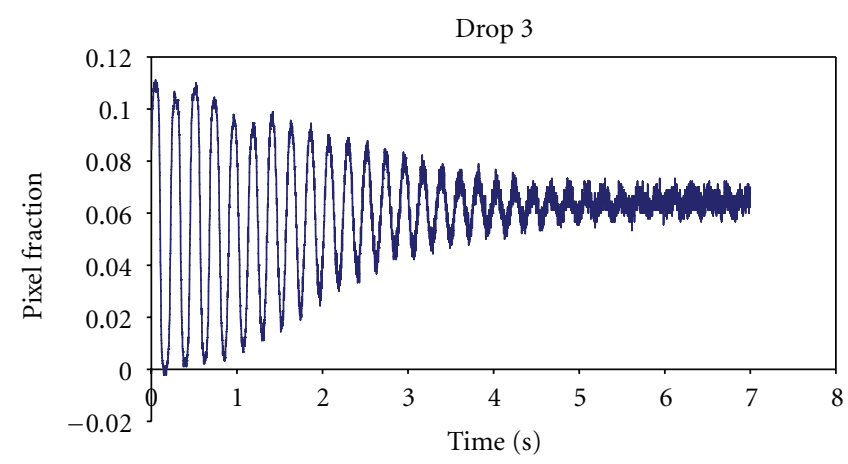

(c)

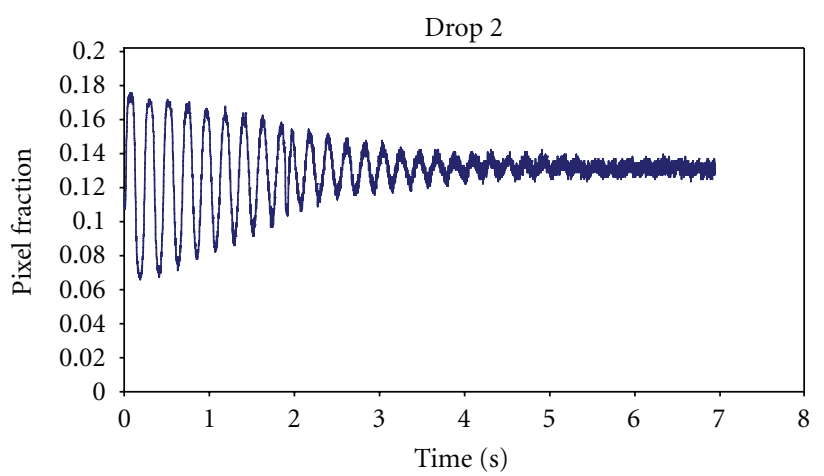

(b)

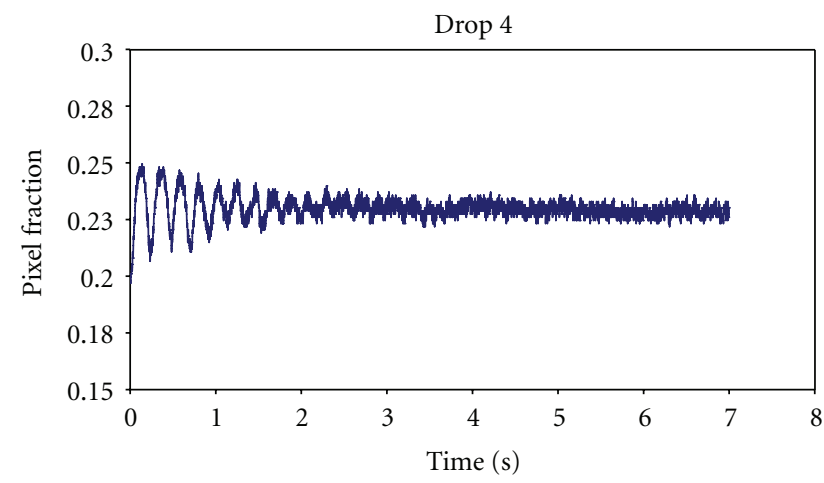

(d)

FIGURE 3: The graphs show the underdamped decay attained for the fraction of the $i$-pixel occupied by the beam used for the four Montagnole drops.

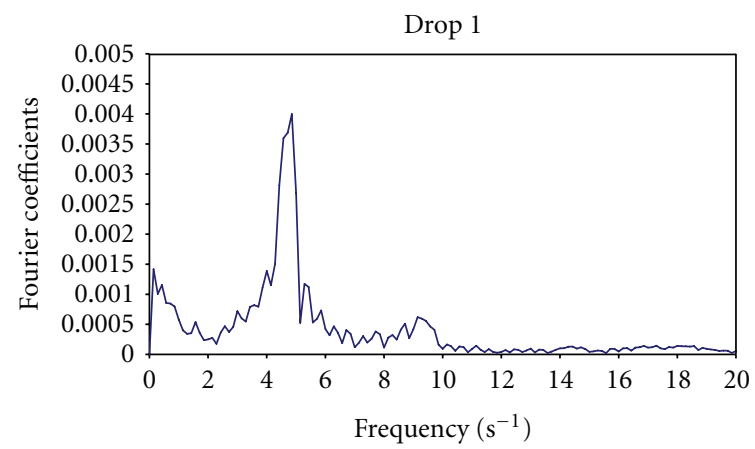

(a)

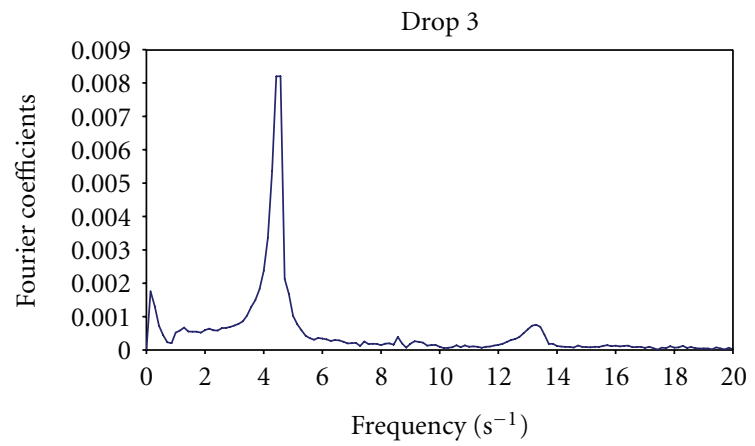

(c)

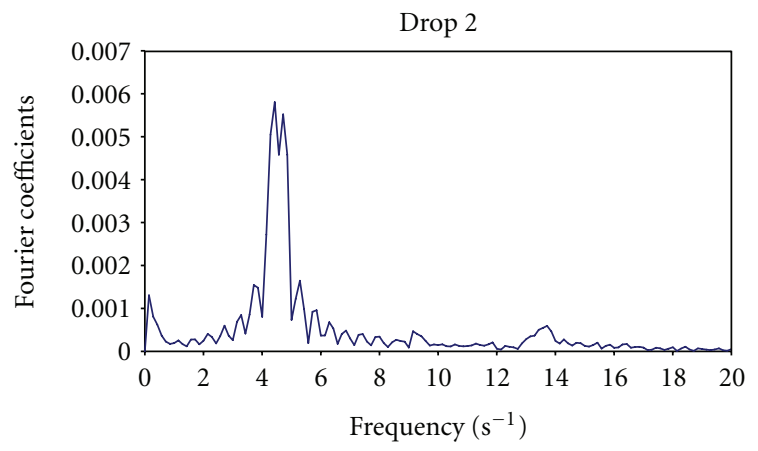

(b)

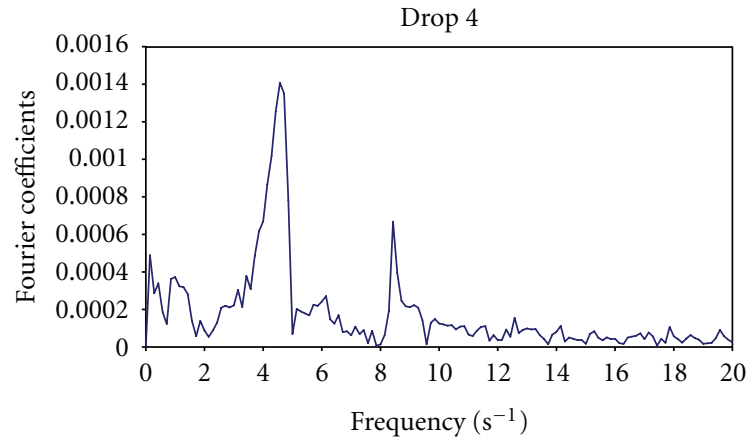

(d)

FIGURE 4: FFT absolute coefficients relative to the $c_{2}$ oscillations. 


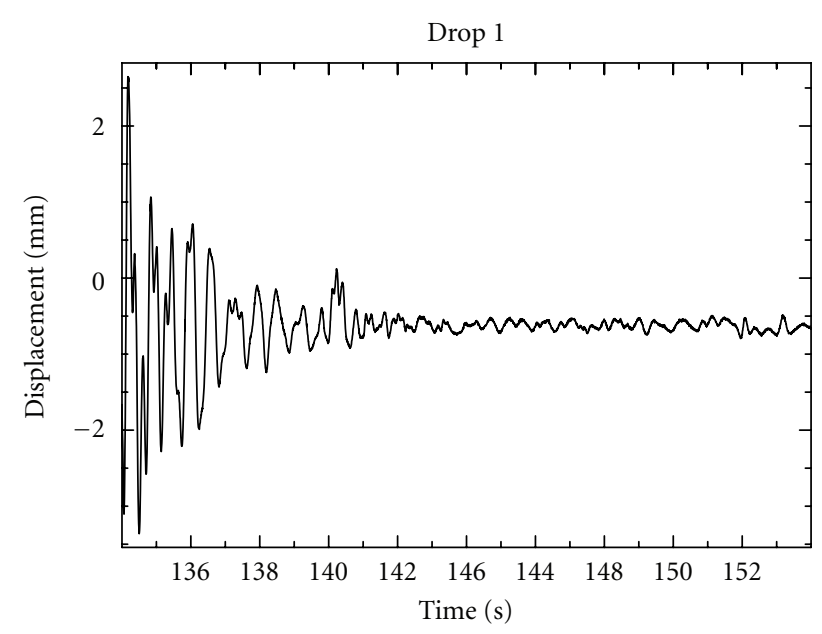

(a)

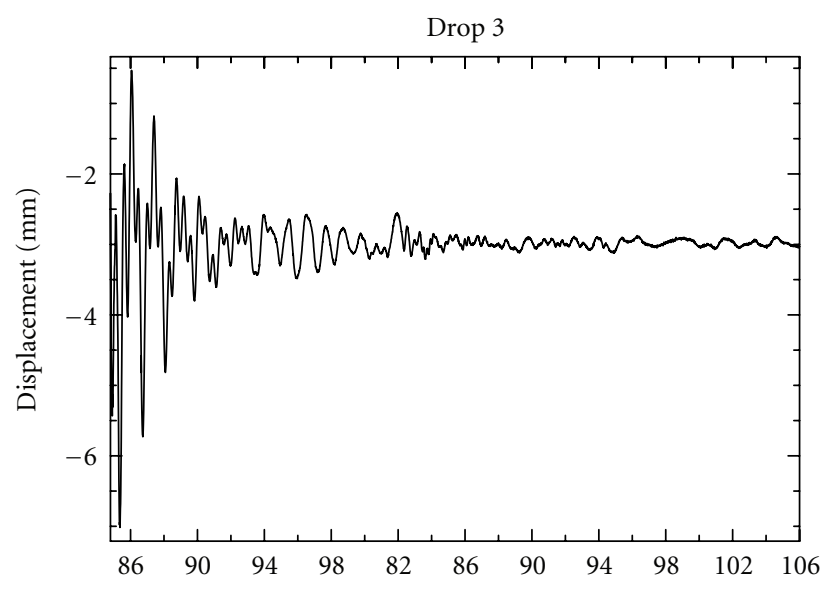

(c)

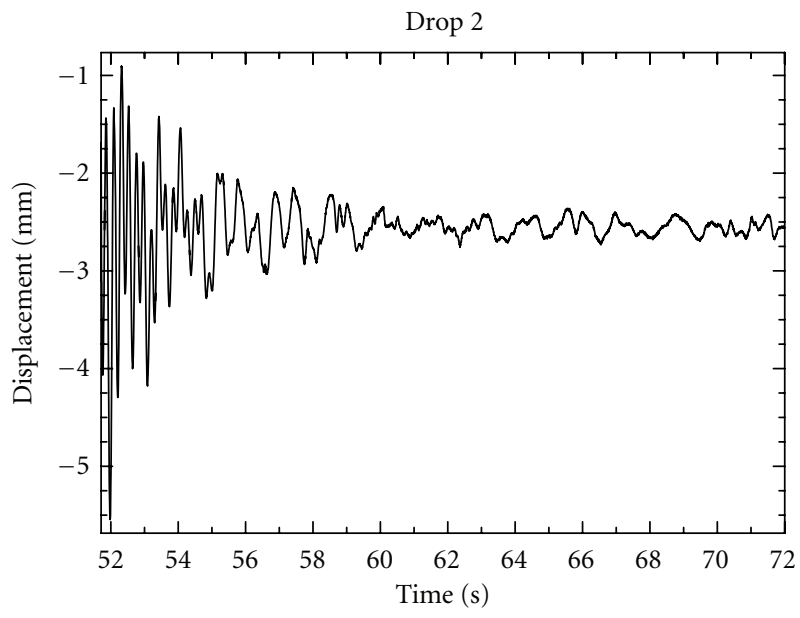

(b)

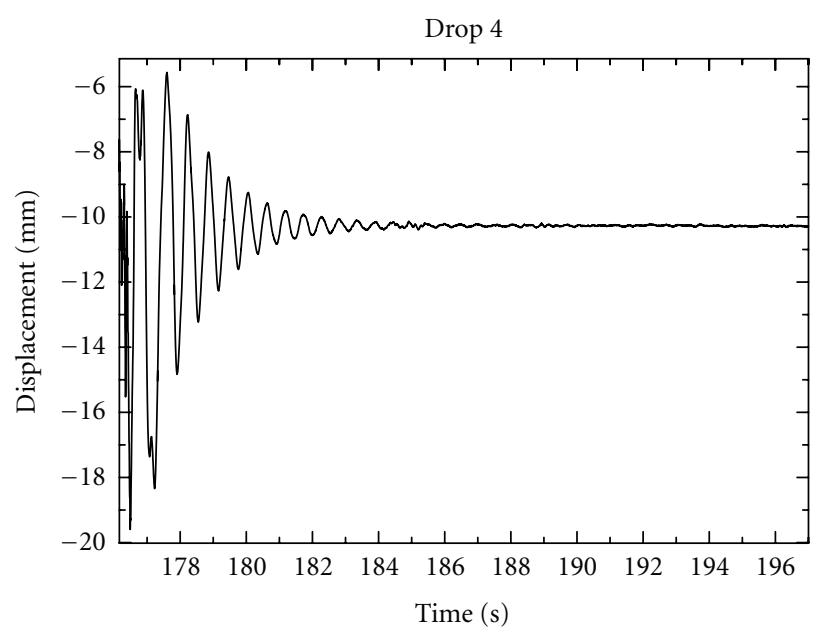

(d)

Figure 5: The graphs show the time domain response of the beam after each drop. A time window of about $20 \mathrm{~s}$ was considered.

TABle 1: Parameters used for calculating the underdamped harmonic oscillator from (5).

\begin{tabular}{lc}
\hline$A_{0}$ & 0.700 \\
\hline$\varphi$ & 1.1 \\
$\omega_{0}$ & 28.049 \\
$\zeta$ & 0.021 \\
Freq. & $4.5 \mathrm{~Hz}$ \\
\hline
\end{tabular}

3.2. Ground-Based Microwave Radar Interferometer Results. IBIS-S data acquired in Montagnole test site were processed by using the commercial IBIS DATA VIEWER (IBISDV) software. The software works by using MATLAB libraries and is based on (i) Inverse Discrete Fourier Transform (IDFT), and (ii) Differential Interferometric technique.

The IBIS-S results allowed the measure of the dynamic response in time and frequency domain of the beam affected by the direct impact of the heavy iron ball.

Figures 5 and 6 show the response in time and frequency domain of the beam oscillation, due to the direct
Table 2: Significant oscillation frequencies of the beam under the four direct impacts.

\begin{tabular}{lcc}
\hline Drop & First frequency $(\mathrm{Hz})$ & Secondary frequency $(\mathrm{Hz})$ \\
\hline 1 & 4.7 & 9.3 \\
2 & 4.5 & 13.7 \\
3 & 4.5 & 13.2 \\
4 & 4.6 & 8.4 \\
\hline
\end{tabular}

impact of the heavy iron ball, for the four drops, respectively.

In the time domain a window of about $20 \mathrm{sec}$ after each direct impact was considered in order to study the dynamic behaviour of the beam. The results show a negative line of sight (LOS) permanent displacement for each drop. In particular, the displacement increases from the first to the last drop as reported in Table 3.

The different LOS permanent displacement could be due to a combination between horizontal and vertical movement of the beam towards the IBIS-S sensor after each drop. This 


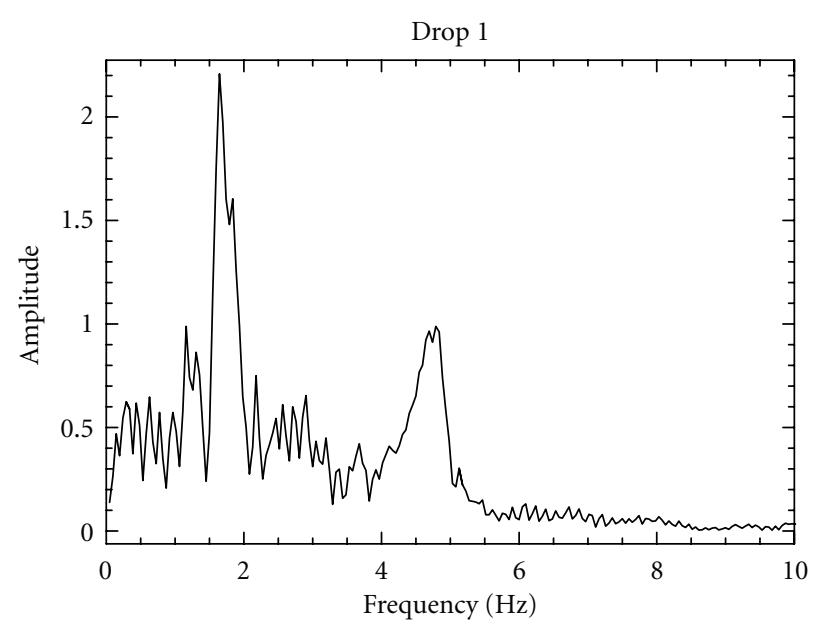

(a)

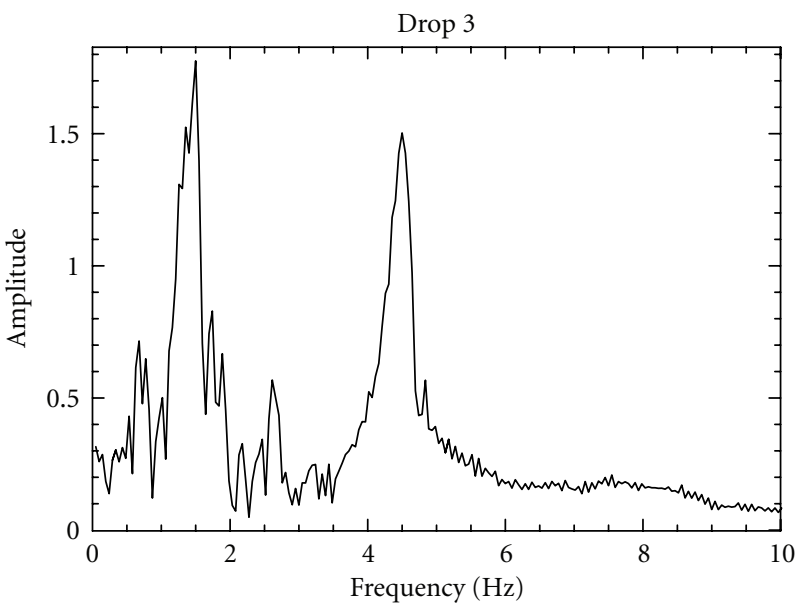

(c)

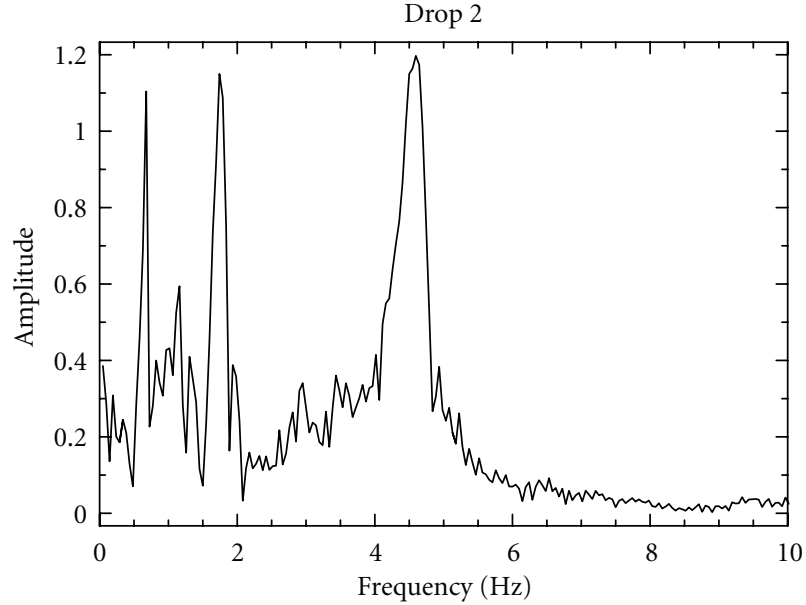

(b)

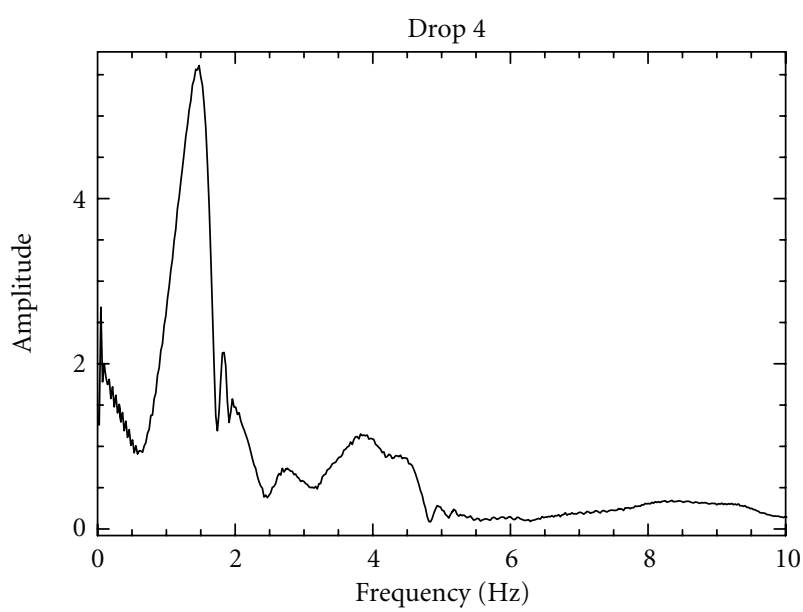

(d)

Figure 6: The graphs report the frequency domain response of the beam during the four drops, a frequency range up to $10 \mathrm{~Hz}$ is considered.

TABLE 3: LOS permanent displacements of the beam after each drop.

\begin{tabular}{lc}
\hline Drop number & Permanent displacement $(\mathrm{mm})$ \\
\hline 1 & -0.5 \\
2 & -2.5 \\
3 & -3.0 \\
4 & -10.0 \\
\hline
\end{tabular}

displacement is less than $3 \mathrm{~mm}$ and grows slightly for the first three drops where the falling block conditions (drop height equal to $1 \mathrm{~m}$ ) are the same. The permanent displacement is amplified after the fourth drop where the iron ball falls from $5 \mathrm{~m}$ height.

Before applying the Fourier Transform to the signals of Figure 5, the mean value and the linear trend were removed from the signal in the selected window. The amplitude of the spectra in a frequency range up to $10 \mathrm{~Hz}$ is reported in Figure 6. For all the drops, two main resonant peak frequencies arise, the first one at about $1.5 \mathrm{~Hz}$ and the second one at about $4.5 \mathrm{~Hz}$. As can be argued from Table 4, both
TABLE 4: Oscillation frequencies of the beam under the four direct impacts measured by Ground-based microwave radar interferometer.

\begin{tabular}{lcc}
\hline Drop & First frequency $(\mathrm{Hz})$ & Secondary frequency $(\mathrm{Hz})$ \\
\hline 1 & 1.6 & 4.8 \\
2 & 1.6 & 4.6 \\
3 & 1.4 & 4.5 \\
4 & 1.4 & 3.8 \\
\hline
\end{tabular}

the main resonant peak frequencies decrease after any direct impact of the iron ball on the beam.

\section{Conclusions}

The proposed multitechniques approach, which is the main aim of the ISTIMES Project, can be useful to individuate suitable parameters which will allow a continuous and/or rapid remote control of the condition of infrastructures to plan appropriate interventions for their conservation. This is even more important when analyzing complex structures 
TABLE 5: Comparison between the oscillation frequencies measured by HFI and GB microwave Radar with the relative percentage difference.

\begin{tabular}{lccc}
\hline Drop & HFI (HZ) & $\begin{array}{c}\text { GB } \\
\text { microwave } \\
\text { radar (Hz) }\end{array}$ & $\begin{array}{c}\text { Relative percentage } \\
\text { difference }\end{array}$ \\
\hline 1 & 4.7 & 4.8 & 2.1 \\
2 & 4.5 & 4.6 & 2.2 \\
3 & 4.5 & 4.5 & 0.0 \\
4 & 4.6 & 3.8 & 17.4 \\
\hline
\end{tabular}

where the use of a single diagnostic tool can provide results leading to ambiguous interpretations.

However, it is difficult to have at disposal a complete setup of instruments measuring the same parameter, for example, as in this case where HFI thermal imagery and ground-based (G-B) microwave radar were applied to study the dynamic behaviour of a beam affected by changeable strains.

G-B microwave radar was applied with the aim of measuring the dynamic displacement of the beam. This technique provided good results, giving information about the behaviour of the target investigated in time and frequency domains. In particular, it allowed the estimation of the displacement affecting the beam after each drops and the measurement of the main frequencies characterizing the structure. Two main peaks at different frequencies were highlighted; both the frequencies decrease after the two last drops due to the increase of the number of severe cracks observed on the beam. The cracks damaging the beam have induced a loss of stiffness with a consequent raise of the period of the eigen modes and a decrease of the eigen-frequencies.

HFI thermal imagery camera was applied with the aim of measuring the structure oscillations by high frequency analysis. Also in this case, the results allowed to discriminate two main peaks at different frequencies after each drop. The first peak measured by HFI at around $4.5 \mathrm{~Hz}$ is comparable with the second peak measured by G-B radar. However, HFI frequencies do not highlight a decrease after the last drop as well as the G-B results (see Table 5). This could be explained considering the different line of view of the two techniques; indeed, G-B microwave radar was placed at a different height respect to the HFI camera and it observed the beam by a specific angle. HFI thermal camera was placed at the same height of the beam and it observed the target perpendicularly. Moreover, the information provided by G$\mathrm{B}$ microwave radar is related to displacement of the beam along the line of sight, while the information from HFI camera is related to the displacement of the beam in the plane perpendicular to the sensor. The comparison between HFI and $\mathrm{G}-\mathrm{B}$ results also suggests that $\mathrm{G}-\mathrm{B}$ radar better points out the low peak frequencies, whereas the HFI thermal camera highlightes the high frequencies.

About the lowest frequencies retrieved by G-B radar (see Table 4), two possible explanation could be given as: a vibration mode of the beam or the main oscillation frequency of dropping machinery after each drop.
Future work will be focused on (a) laboratory and field measurements to better understand the different results attained by the HFI and GB microwave Radar (b) a numerical modelling of the beam to estimate its main frequencies, and (c) the development and analysis of the integration of the data acquired by different sensor technologies on infrastructures in order to evaluate their effectiveness and suitability for this kind of application.

\section{Acknowledgment}

The research leading to these results has received funding from the European Community's Seventh Framework Programme (FP7/2007-2013) under Grant Agreement no. 225663 Joint Call FP7-ICT-SEC-2007-1.

\section{References}

[1] C. Kohl, M. Krause, C. Maierhofer, K. Mayer, J. Wöstmann, and H. Wiggenhauser, "3D-visualisation of NDT data using a data fusion technique," Insight, vol. 45, no. 12, pp. 800-804, 2003.

[2] Ch. Maierhofer, R. Arndt, M. Röllig et al., "Application of impulse-thermography for non-destructive assessment of concrete structures," Cement and Concrete Composites, vol. 28, no. 4, pp. 393-401, 2006.

[3] M. Proto, M. Bavusi, R. Bernini et al., "Transport infrastructure surveillance and monitoring by electromagnetic sensing: the ISTIMES project," Sensors, vol. 10, no. 12, pp. 1062010639, 2010.

[4] S. Rödelsperger, G. Läufer, C. Gerstenecker, and M. Becker, "Monitoring of displacements with ground-based microwave interferometry: IBIS-S and IBIS-L," Journal of Applied Geodesy, vol. 4, no. 1, pp. 41-54, 2010.

[5] J. R. Brown and H. R. Hamilton, "Quantitative infrared thermography inspection for FRP applied to concrete using single pixel analysis," Construction and Building Materials. In press.

[6] S. Pascucci, C. Bassani, A. Palombo, M. Poscolieri, and R. Cavalli, "Road asphalt pavements analyzed by airborne thermal remote sensing: preliminary results of the venice highway," Sensors, vol. 8, no. 2, pp. 1278-1296, 2008.

[7] S. W. Doebling, C. R. Farrar, M. B. Prime, and D. Shevitz, Damage identification and health monitoring of structural and mechanical systems from changes in their vibration characteristics: a literature review, Los Alamos National Laboratory, 1996.

[8] C. Gentile and G. Bernardini, "Output-only modal identification of a reinforced concrete bridge from radar-based measurements," NDT and E International, vol. 41, no. 7, pp. 544$553,2008$.

[9] C. R. Farrar, T. W. Darling, A. Migliori, and W. E. Baker, "Microwave interferometers for non-contact vibration measurements on large structures," Mechanical Systems and Signal Processing, vol. 13, no. 2, pp. 241-253, 1999.

[10] M. Pieraccini, F. Parrini, M. Fratini, C. Atzeni, P. Spinelli, and M. Micheloni, "Static and dynamic testing of bridges through microwave interferometry," NDT and E International, vol. 40, no. 3, pp. 208-214, 2007.

[11] M. Pieraccini, M. Fratini, D. Dei, and C. Atzeni, "Structural testing of Historical Heritage Site Towers by microwave remote sensing," Journal of Cultural Heritage, vol. 10, no. 2, pp. 174$182,2009$. 
[12] C. Atzeni, A. Bicci, D. Dei, M. Fratini, and M. Pieraccini, "Remote survey of the leaning tower of pisa by interferometric sensing," IEEE Geoscience and Remote Sensing Letters, vol. 7, no. 1, Article ID 5290014, pp. 185-189, 2010.

[13] C. Gentile, "Application of microwave remote sensing to dynamic testing of stay-cables," Remote Sensing, vol. 2, no. 1, pp. 36-51, 2010.

[14] M. Pieraccini, F. Parrini, M. Fratini, C. Atzeni, and P. Spinelli, "In-service testing of wind turbine towers using a microwave sensor," Renewable Energy, vol. 33, no. 1, pp. 13-21, 2008.

[15] J. D. Taylor, Ed., Ultra-Wideband Radar Technology, CRC Press, 2001.

[16] F. M. Henderson and A. J. Lewis, Eds., Manual of Remote Sensing. Principles and Applications of Imaging Radar, John Wiley \& Sons, New York, NY, USA, 1998.

[17] C. Gentile and G. Bernardini, "An interferometric radar for non-contact measurement of deflections on civil engineering structures: laboratory and full-scale tests," Structure and Infrastructure Engineering, vol. 6, no. 5, pp. 521-534, 2010.

[18] G. Bernardini, G. De Pasquale, A. Bicci et al., "Microwave interferometer for ambient vibration measurement on civil engineering structures: 1. Principles of the radar technique and laboratory tests," in Experimental Vibration Analysis for Civil Engineering Structures (EVACES '07), Porto, Portugal, October 2007.

[19] M. Pieraccini, M. Fratini, F. Parrini, G. Macaluso, and C. Atzeni, "High-speed CW step-frequency coherent radar for dynamic monitoring of civil engineering structures," Electronics Letters, vol. 40, no. 14, pp. 907-908, 2004. 

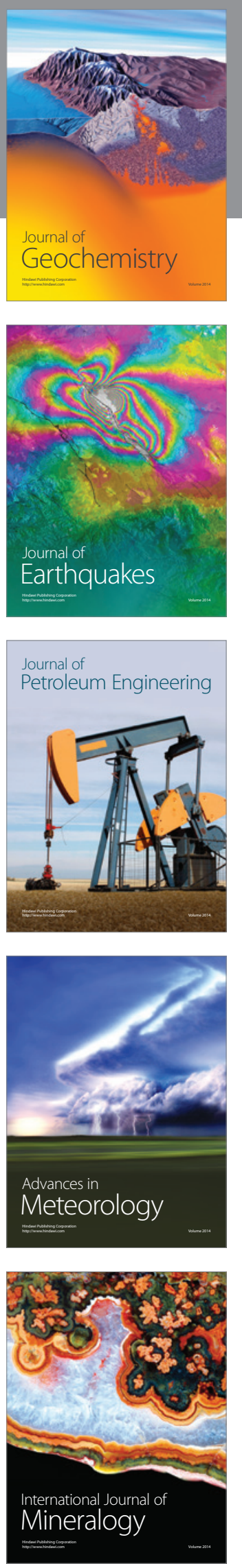
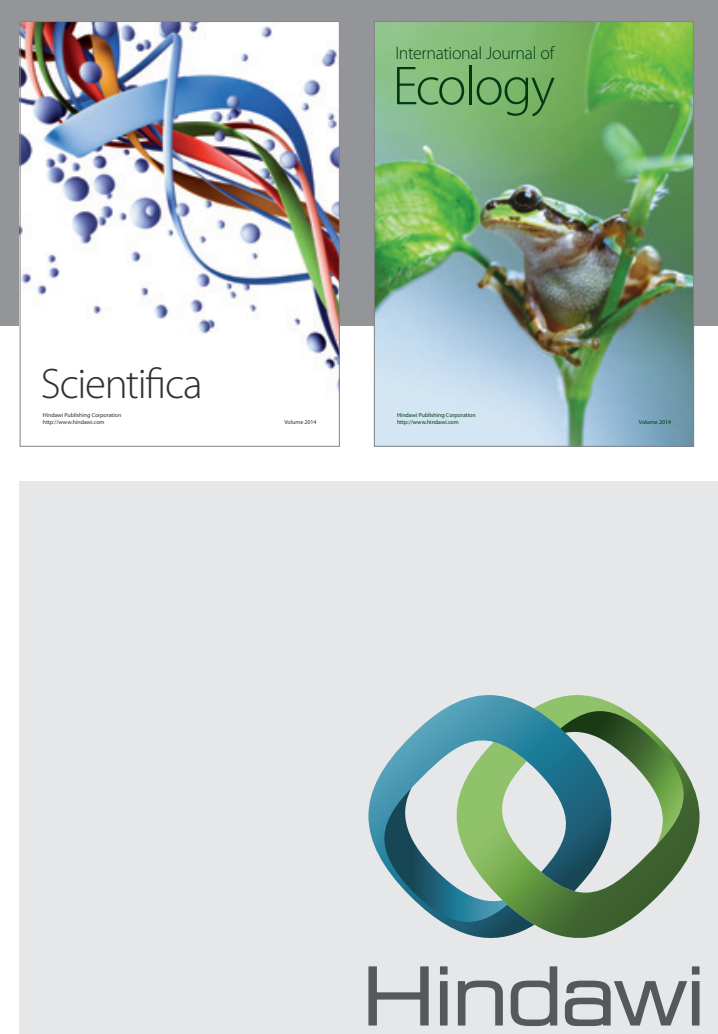

Submit your manuscripts at http://www.hindawi.com
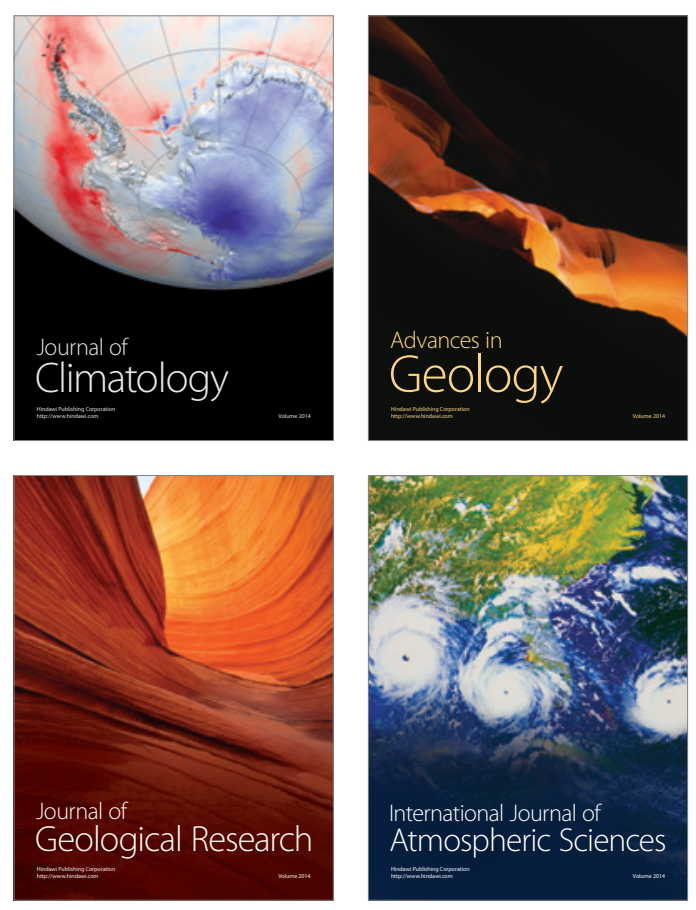
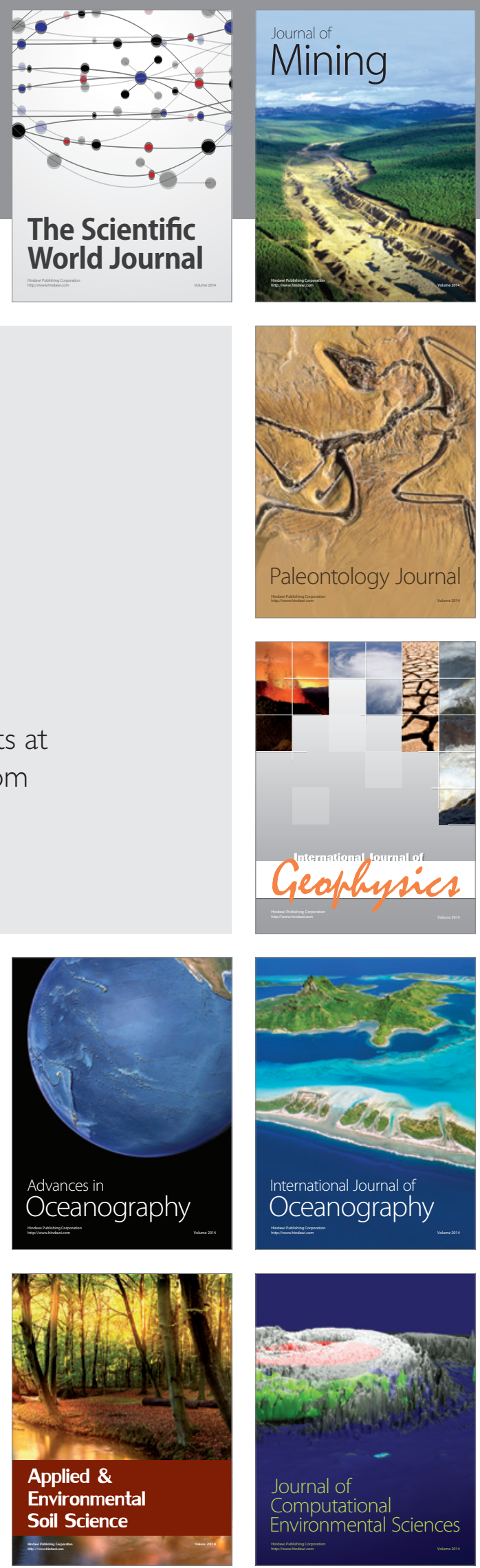